Kohji Itoh · Yasunori Naganawa • Fumiko Matsuzawa

Seiichi Aikawa • Hirofumi Doi • Naokazu Sasagasako

Takeshi Yamada · Jun-ichi Kira • Takuro Kobayashi

Alexey V. Pshezhetsky • Hitoshi Sakuraba

\title{
Novel missense mutations in the human lysosomal sialidase gene in sialidosis patients and prediction of structural alterations of mutant enzymes
}

\begin{abstract}
Three novel missense mutations in the human lysosomal sialidase gene causing amino acid substitutions (P80L, W240R, and P316S) in the coding region were identified in two Japanese sialidosis patients. One patient with a severe, congenital form of type 2 sialidosis was a compound heterozygote for 239C-to-T (P80L) and 718T-toC (W240R). The other patient with a mild juvenile-onset phenotype (type 1) was a homozygote for the base substitution of 946C-to-T (P316S). None of these mutant cDNA products showed enzymatic activity toward an artificial substrate when coexpressed in galactosialidosis fibroblastic cells together with protective protein/cathepsin A (PPCA). All mutants showed a reticular immunofluorescence distribution when coexpressed with the PPCA gene in COS-1 cells, suggesting that the gene products were retained in the endoplasmic reticulum/Golgi area or rapidly degraded in the lysosomes. Homology modeling of the structural changes introduced by the mutations predicted that the P80L and P316S transversions cause large conformational
\end{abstract}

K. $\operatorname{Itoh}^{1}(\bowtie) \cdot$ Y. Naganawa $\cdot$ H. Sakuraba

Department of Clinical Genetics, The Tokyo Metropolitan Institute of Medical Science, Tokyo Metropolitan Organization for Medical

Research, Tokyo, Japan

F. Matsuzawa $\cdot$ S. Aikawa $\cdot$ H. Doi

Celestar Lexico Sciences, Chiba, Japan

N. Sasagasako

Department of Neurology, Hamanomachi Hospital, Federation of Public Services and Affiliated Personal Aid Association, Fukuoka, Japan

T. Yamada $\cdot$ J. Kira $\cdot$ T. Kobayashi ${ }^{\dagger}$

Department of Neurology, Neurological Institute, Graduate School of Medical Sciences, Kyushu University, Fukuoka, Japan ('deceased)

A.V. Pshezhetsky

Service de Génétique Médicale, Départment de Pédiatrie, Hôpital

Sainte-Justine, Université de Montréal, Montréal, Canada

\section{Present address:}

${ }^{1}$ Division of Medicinal Biotechnology, Institute for Medicinal

Resources, Faculty of Pharmaceutical Sciences, University of

Tokushima, 1-78 Sho-machi, Tokushima 770-8505, Japan

Tel. +81-88-633-7290; Fax: +81-88-633-7290

e-mail: kitoh@ph2.tokushima-u.ac.jp changes including the active site residues responsible for binding the sialic acid carboxylate group. The W240R substitution was deduced to influence the molecular surface structure of a limited region of the constructed models, which was also influenced by previously identified V217M and G243R transversions.

Key words Lysosomal sialidase - Sialidosis - Molecular modeling - Protective protein/cathepsin A - Galactosialidosis

\section{Introduction}

Lysosomal sialidase (neuraminidase, EC 3.2.1.18) catalyzes the hydrolysis of the terminal sialic acid residues of sialylated glycoconjugates. In mammals, the enzyme associates with acid $\beta$-galactosidase (EC 3.2.1.23) and protective protein/cathepsin A (PPCA, EC 3.4.16.1) to form a multienzyme complex in lysosomes (d'Azzo et al. 1982; Verheijen et al. 1982, 1985; Hiraiwa et al. 1988; van der Horst et al. 1989). In paticular, association with PPCA is essential for expression of the sialidase activity (d'Azzo et al. 1982; van der Spoel et al. 1998). Sialidosis is an autosomal recessive metabolic inborn error caused by a genetic defect of lysosomal sialidase, which is accompanied by accumulation in tissues and urinary excretion of sialyloligosaccharides (reviewed by Lowden and O'Brien 1979; Thomas 2000). The clinical phenotypes of sialidosis are classified in two major groups according to the age of onset and severity: late-onset type 1 sialidosis is a relatively mild form characterized by macular cherry-red spots and myoclonus, and early infantile-onset type 2 sialidosis is a severe condition distinguished by the appearance of dysmorphic manifestations in addition to neurological symptoms and cherry-red spots. Sialidosis type 2 is further divided into three clinical subtypes: congenital, infantile, and juvenile. The congenital form is the most severe subtype, and is associated with hydrops fetalis, neonatal ascites, edema, inguinal hernia, hepatosplenomegaly, and death at an early 
age. Another human sialidase deficiency, galactosialidosis, is caused by a primary defect of PPCA with combined decreases in lysosomal sialidase, $\beta$-galactosidase, and cathepsin A activities, and has clinical manifestations similar to those of sialidosis (reviewed by d'Azzo et al. 2000).

In recent years, the human lysosomal sialidase gene has been cloned (Bonten et al. 1996; Pshezhetsky et al. 1997; Milner et al. 1997) on the basis of the genomic and structural information on the sialidases obtained from a variety of species, including viruses and bacteria (Roggentin et al. 1989; Rothe et al. 1991; Crennell et al. 1993, 1994; Gaskell et al. 1995). The human sialidase cDNA contains a $1.2-\mathrm{kb}$ open reading frame and a polyadenylation site within the $3^{\prime}$ untranslated region. The deduced amino acid sequence contains four Asp boxes (S/T-X-D-S-G-X-X-W/F), which are repeated at topologically equivalent positions, and a (F)RIP sequence motif, which is part of the active site. In addition, the sequence revealed the presence of three potential $\mathrm{N}$-glycosylation sites and a carboxy-terminal Y-X-Xhydrophobic residue motif similar to that found in other lysosomal integral membrane proteins (Guarnieri et al. 1993; Sosa et al. 1993). A number of mutations have been identified in sialidosis patients (Bonten et al. 1996; Bonten and d'Azzo 2000; Pshezhetsky et al. 1997; Lukong et al. 2000; Naganawa et al. 2000).

Conservation of the basic structural fold of sialidases, which consists of six four-stranded antiparallel $\beta$-sheets arranged as the blades of a propeller around an axis of symmetry passing through the active site, among various species has also been shown (Crennell et al. 1993, 1994; Gaskell et al. 1995), which makes it possible to model the tertiary structure of human lysosomal sialidase (Lukong et al. 2000; Naganawa et al. 2000). In the present study, we identified three new missense mutations resulting in amino acid substitutions, and characterized the mutant products biochemically. Using homology modeling, we deduced the structural changes in the structure of the sialidase molecule caused by the substitutions.

\section{Subjects and methods}

\section{Subjects}

Sialidosis patients were diagnosed biochemically by assaying lysosomal enzymes (Suzuki et al. 1981; Suzuki 1987) in cultured skin fibroblasts or amniocytes. All fibroblastic cell strains showed the isolated sialidase deficiency. Other enzyme activities, including those of $\beta$-galactosidase and cathepsin $\mathrm{A}$, were normal.

The research was carried out in accordance with the Declaration of Helsinki of the World Medical Association, and was approved by the ethical committee of the institution in which the work was performed. The subjects or their families gave informed consent to the work.

Patient 1. A male patient was prenatally diagnosed in the 21st week of gestation during a fifth pregnancy following the nonconsanguineous parents' request, as previously described (Sasagasako et al. 1993). The proband of the family was the second female child of the parents, who was found to have hydrops fetalis in the 33rd gestational week. At birth, severe subcutaneous edema, pleural effusion, ascites, and hepatosplenomegaly were observed. She was diagnosed as having a congenital form of type 2 sialidosis, and she subsequently developed respiratory failure and died 87 days after birth. The male fetus was artificially aborted in the 21st gestational week. A pathological study revealed slight generalized subcutaneous edema, mild pleural effusion, ascites, and mild hepatosplenomegaly. Light microscopic analysis disclosed a large number of vacuoles in the cytoplasm of the liver, spleen, skin, bone marrow, lymph nodes, adrenal cortex, anterior horn cells of the spinal cord, and neuronal cells in the cerebrum.

Patient 2. The other patient is the 24-year-old male offspring of consanguineous parents. At the age of 14 years, he developed gait disturbance and dysgraphia. Generalized convulsive seizure attacks, visual disturbance, dysarthria, and action myoclonus were also observed. Physical examination at 18 years of age revealed bilateral cherry-red spots and cerebellar dysfunction. He was diagnosed as having type 1 sialidosis. The progression of the symptoms was rapid. Difficulty in swallowing appeared by 18 years of age, and he suffered from recurrent bronchopneumonia. His elder brother died of sialidosis at 18 years of age, but detailed clinical data are not available.

\section{Cell culture}

Human skin fibroblasts derived from the two patients with sialidosis were maintained in Ham's F-10 medium supplemented with $10 \%$ fetal calf serum (FCS) and antibiotics. For transient expression of the wild-type and mutant sialidase cDNAs, a fibroblastic cell line derived from a galactosialidosis patient (ASVGS-1) (Shimmoto et al. 1993) or COS-1 cells (Gluzman 1988) was cultured in the same medium.

\section{Gene analysis}

Total RNA and genomic DNA were isolated from the cultured fibroblasts as described previously (Davis et al. 1986). Lysosomal sialidase cDNA, including the entire coding region, was obtained by reverse transcription (RT)polymerase chain reaction (PCR), and the nucleotide sequence was determined according to a previous report (Naganawa et al. 2000). As described in the Results section, 239C-to-T (P80L), 718T-to-C (W240R), and 946C-to-T (P316S) substitutions were detected in exons 2, 4, and 5, respectively, of the lysosomal sialidase gene. To confirm the presence of these mutations, genomic DNA fragments including exons 2,4 , and 5 were amplified by means of PCR using sets of primers: sense, 5'-CTTTGTTGACCCTTC CTC-3', and antisense, 5'-GGCTCTTTCACCCAGAC ATC-3', for exon 2; sense, 5'-TGAGCCCCTAGAGTCT 
CC-3', and antisense, 5'-AGTCCTGAAGGCAGAATA CC-3' ${ }^{\prime}$, for exon 4; and sense, $5^{\prime}$-CTCAGTGCCACACAC CCTTG-3', and antisense, 5'-TCTAGACACAGGGCTC TC-3', for exon 5. The amplified DNA fragments were analyzed by direct sequencing.

\section{Transient expression of lysosomal sialidase cDNA}

For expression of the mutant lysosomal sialidase cDNA, the DNA fragment obtained from the mutant cDNA subclone, containing 239C-to-T (P80L), 718T-to-C (W240R), or 946C-to-T (P316S), was prepared by double-digestion with $B s t$ EII and $K p n I$, and substituted for the pCMV (Clontech, PaloAlto, CA, USA)-sialidase (Sial) plasmid, which contains the full-length human wild-type lysosomal sialidase cDNA. The cloned plasmids were designated as pCMV-Sial (P80L), pCMV-Sial (W240R), and pCMV-Sial (P316S), respectively. The final constructs were verified by sequencing.

ASVGS- 1 and COS- 1 cells were seeded onto $60-\mathrm{mm}$ dishes $\left(2 \times 10^{5}\right.$ cells $)$ for the enzyme assay $24 \mathrm{~h}$ before transfection. For immunocytochemical analysis, COS-1 cells were seeded onto two-well chamber slides (Lab-Tek; Nunc, Naperville, IL, USA) at a density of $1 \times 10^{5}$ cells per well. The transfection was performed using DMRIE-C reagent (GIBCO/BRL, Rockville, MD, USA) according to the manufacturer's protocol. Briefly, a mixture comprising the plasmid DNA $(5 \mu \mathrm{g})$ and DMRIE-C reagent $(10 \mu \mathrm{l})$ was added to cells cultured in FCS-free Ham's F-10 medium. After $5 \mathrm{~h}$ incubation, the culture medium was replaced with Ham's F-10 medium containing 10\% FCS, and cells were cultured for 4 more days. The $\mathrm{pCXN}_{2}$ vector containing human wild-type PPCA cDNA ( $\mathrm{pCXN}_{2}-\mathrm{PP}, 5 \mu \mathrm{g}$ ) (Shimmoto et al. 1993) was cotransfected with the wild-type or mutant lysosomal sialidase cDNA, which is necessary for the activation of the latter enzyme.

\section{Enzyme assay}

Transfected ASVGS-1 cells were washed with phosphatebuffered saline (PBS), harvested by scraping, and then suspended in distilled water containing $0.1 \mathrm{mM}$ leupeptin. A homogenate was prepared by pipetting, and then sialidase activity was measured immediately according to the method reported previously (Suzuki et al. 1981). The rest of the homogenate was sonicated and used for other lysosomal enzyme assays and protein determination. Glycosidase activities, including those of sialidase, $\beta$-galactosidase and $\beta$-hexosaminidase, were assayed fluorometrically using 4methylumbelliferyl glycosides as a substrate (Suzuki 1987). Cathepsin A activity was measured at pH 5.6, using Z-PheLeu as a substrate (Itoh et al. 1991). Protein determination was performed with a $D C$ assay kit (Bio-Rad, Richmond, CA, USA), using bovine serum albumin (BSA) as a standard.

Immunocytochemical analysis

Transfected COS-1 cells were fixed with ice-cold $4 \%$ paraformaldehyde/PBS (v/v) and then $80 \%$ methanol/PBS (v/v). After nonspecific binding was blocked with 5\% goat serum and $1 \%$ BSA in PBS, they were treated with the antisialidase antibody (Vinogradova et al. 1998) overnight at $4^{\circ} \mathrm{C}$. Then, the cells were washed with PBS, followed by treatment with fluorescein isothiocyanate (FITC)-labeled $\mathrm{F}\left(\mathrm{ab}^{\prime}\right)_{2}$ fragments of goat anti-rabbit IgG (BioSource, Camarillo, CA, USA). After washing with PBS, the cells were mounted with $55 \%$ glycerol/PBS (v/v) containing $3.8 \%$ diazabicyclo(2,2,2)octane (DABCO). The stained cells were examined under a fluorescence microscope (Axiophot; Zeiss, Oberkochen, Germany).

Modeling of human wild-type and mutant lysosomal sialidase structures

Structural models of human wild-type lysosomal sialidase and its mutants were built using the atomic coordinates of homologous sialidases in the Protein Data Bank (PDB; Brookhaven National Laboratory, Upton, NY, USA) (Sussman et al. 1998) and molecular modeling software, SYBYL/COMPOSER (TRIPOS, Mountain View, CA, USA). As templates, the structures of the homologous sialidases from Salmonella typhimurium LT2 (PDB code; 2SIL) (Crennel et al. 1993), Micromonospora viridifaciens (PDB code; 1EUR) (Gaskell et al. 1995), and Vibrio cholerae (PDB code; 1KIT) (Crennell et al. 1994) were used. The template structures were superimposed by aligning the active site residues to determine structurally conserved regions (SCRs). Then, the sequence of human sialidase was aligned with the sequences of SCRs manually. A backbone of human sialidase corresponding to each SCR was built by fitting a fragment from one of the homologues, and a rule-based procedure was used to determine the sidechain conformations (Sutcliffe et al. 1987; Burmeister et al. 1992). Loop regions were constructed with fragments selected from the protein substructure database (Jones and Thirup 1986; Claessens et al. 1989). An energy minimization procedure was performed to optimize conformations and side-chain rotamers. Mutant models were constructed in the same way as the wild-type model. To evaluate the influence of the mutations (P80L, V217M, W240R, G234R and P316S), each mutant model was superimposed on the wildtype model by aligning the $\mathrm{C} \alpha$ atoms by means of the leastsquare-mean fitting method (Kabsh 1976, 1978).

\section{Results}

Gene analysis

For gene mutation analysis, the RT-PCR products of the entire cDNA coding sequence for lysosomal sialidase were subcloned and sequenced. We found that three types of cDNA clones were derived from two unrelated Japanese sialidosis patients. Each clone contained a single base substitution when compared with the reported wild-type sialidase sequence: 239C-to-T (P80L), 718T-to-C (W240R), 
and 946C-to-T (P316S) (data not shown). As these base changes are located in exons 2,4 , and 5 , respectively, genomic DNA fragments corresponding to these exons were amplified by PCR and then directly sequenced. The results revealed that the patient with the congenital form of type 2 sialidosis was heterozygous for the 239C-to-T (P80L) and 718T-to-C (W240R) substitutions. The other type 1 patient was homozygous for the 946C-to-T (P316S) transition.

\section{Transient gene expression analysis}

To characterize the effects of the amino acid substitutions P80L, W240R, and P316S on sialidase activity, the wild-type or mutant sialidase cDNAs were transiently co-expressed with the human PPCA cDNA in a galactosialidosis-derived fibroblastic cell line (ASVGS-1 cells). The expression was also previously performed for a mutant with 649G-to-A (V217M) substitution (Naganawa et al. 2000). The results are summarized in Table 1. In ASVGS-1 cells, sialidase activity as well as $\beta$-galactosidase and cathepsin A activities are markedly decreased because of a genetic defect of PPCA, as mentioned above. Mock transfection with the human PPCA cDNA alone restored the sialidase, $\beta$ galactosidase, and cathepsin $\mathrm{A}$ activities in the cells. Cotransfection of the wild-type sialidase cDNA with $P P C A$ caused an approximately five-fold increase in sialidase activity compared with that of the mock transfection. $\beta$ Hexosaminidase activity, used as a reference, did not change significantly. However, cotransfection of the mutant cDNAs containing the 239C-to-T (P80L), 718T-to-C (W240R), and 946C-to-T (P316S) substitutions did not cause any increase in sialidase activity compared with that of the mock transfection. In contrast, the expression of the 649G-to-A (V217M) mutant cDNA restored the enzyme activity to about $30 \%$ that of the ASVGS-1 cells cotransfected with the wild-type sialidase and PPCA cDNAs, as previously reported (Naganawa et al. 2000).

Figure 1 shows the results of an immunocytochemical analysis performed with an anti-sialidase antibody. When COS cells were cotransfected with the wild-type sialidase cDNA and PPCA cDNA, typical lysosomal granular staining was observed (Fig. 1A). In contrast, the gene products with the P80L, W240R, and P316S substitutions showed only a reticular staining pattern, a characteristic of the endoplasmic reticulum/Golgi apparatus (Figs. 1B, C, D, respectively). On the other hand, both perinuclear reticular and partial punctate cytoplasmic staining were observed as a result of the expression of the V217M mutant (Fig. 1E).

Structural modeling of the wild-type and mutant lysosomal sialidases

To locate the positions of amino acid substitutions and to predict their potential effects on the tertiary structure, we built structural models of the human wild-type and mutant lysosomal sialidases using the atomic coordinates of homologous sialidases from Salmonella typhimurium LT2 (Crennell et al. 1993), Micromonospora viridifaciens (Gaskell et al. 1995), and Vibrio cholerae (Crennell et al. 1994) as templates. As shown in Fig. 2, the deduced structure of wild-type sialidase shows a barrel-like fold composed of six four-stranded antiparallel $\beta$-sheet units with the predicted active site cave in the center. This six-bladed propeller-like shape is shared between bacterial and viral sialidases (Burmeister et al. 1992; Crennell et al. 1993, 1994; Gaskell et al. 1995). Each $\beta$-sheet unit has loop regions connecting the second strand to the third and the first strand to the fourth. The amino acid residues comprising the putative active center (R78, R97, D103, D135, S156, E264, R280, Q282, R341, Y370, and E394) are located in these loop regions on the upper side of the barrel structure (Fig. 2). In contrast, the conserved Asp-box domains, connecting the third to the fourth strand of each $\beta$-sheet unit, are located on the lower side of the barrel structure. The model also shows the positions of the mutations (P80L, P316S, W240R, G243R, and V217M) identified in our present and previous studies (Naganawa et al. 2000) (Fig. 2).

Further, we constructed structural models of the mutant enzymes and compared them with that of the wild-type. As a result, the mutations could be classified into two groups on the basis of their effects on the tertiary structure. The mutation group (P80L and P316S) caused large conformational changes, including in the active center, and the mutations from the other group (W240R, G243R, and V217M) caused local structural changes in a limited molecular surface region, as shown in Figs. 3 and 4.

Table 1. Transient expression of lysosomal sialidase cDNA

\begin{tabular}{lcccc}
\hline Plasmids & $\begin{array}{l}\text { Sialidase } \\
(\mathrm{nmol} / \mathrm{h} / \mathrm{mg} \text { protein })\end{array}$ & $\begin{array}{l}\text { Cathepsin A } \\
(\mu \mathrm{mol} / \mathrm{h} / \mathrm{mg} \text { protein })\end{array}$ & $\begin{array}{l}\beta \text {-Galactosidase } \\
(\mathrm{nmol} / \mathrm{h} / \mathrm{mg} \text { protein })\end{array}$ & $\begin{array}{l}\beta \text {-Hexosaminidase } \\
(\mu \mathrm{mol} / \mathrm{h} / \mathrm{mg} \text { protein })\end{array}$ \\
\hline None & $<1$ & $<0.1$ & 20 & 3.6 \\
pCXN & -PP & 2.0 & 52 & 2.6 \\
pCMV-Sial and pCXN ${ }_{2}$-PP & 147 & 1.9 & 54 & 2.4 \\
pCMV-Sial (P80L) and pCXN ${ }_{2}$-PP & 33 & 2.0 & 64 & 3.0 \\
pCMV-Sial (W240R) and $\mathrm{pCXN}_{2}$-PP & 32 & 1.8 & 63 & 2.9 \\
pCMV-Sial (P316S) 28 and $\mathrm{pCXN}_{2}$-PP & 28 & 1.4 & 55 & 2.9 \\
pCMV-Sial (V217M) and pCXN 2 -PP & 66 & 2.0 & 55 & 2.6
\end{tabular}

Lysosomal enzyme activities in a galactosialidosis fibroblastic cell line (ASVGS-1 cells) transfected with sialidase plasmids, as described under Subjects and methods. Values are the means of three independent measurements 

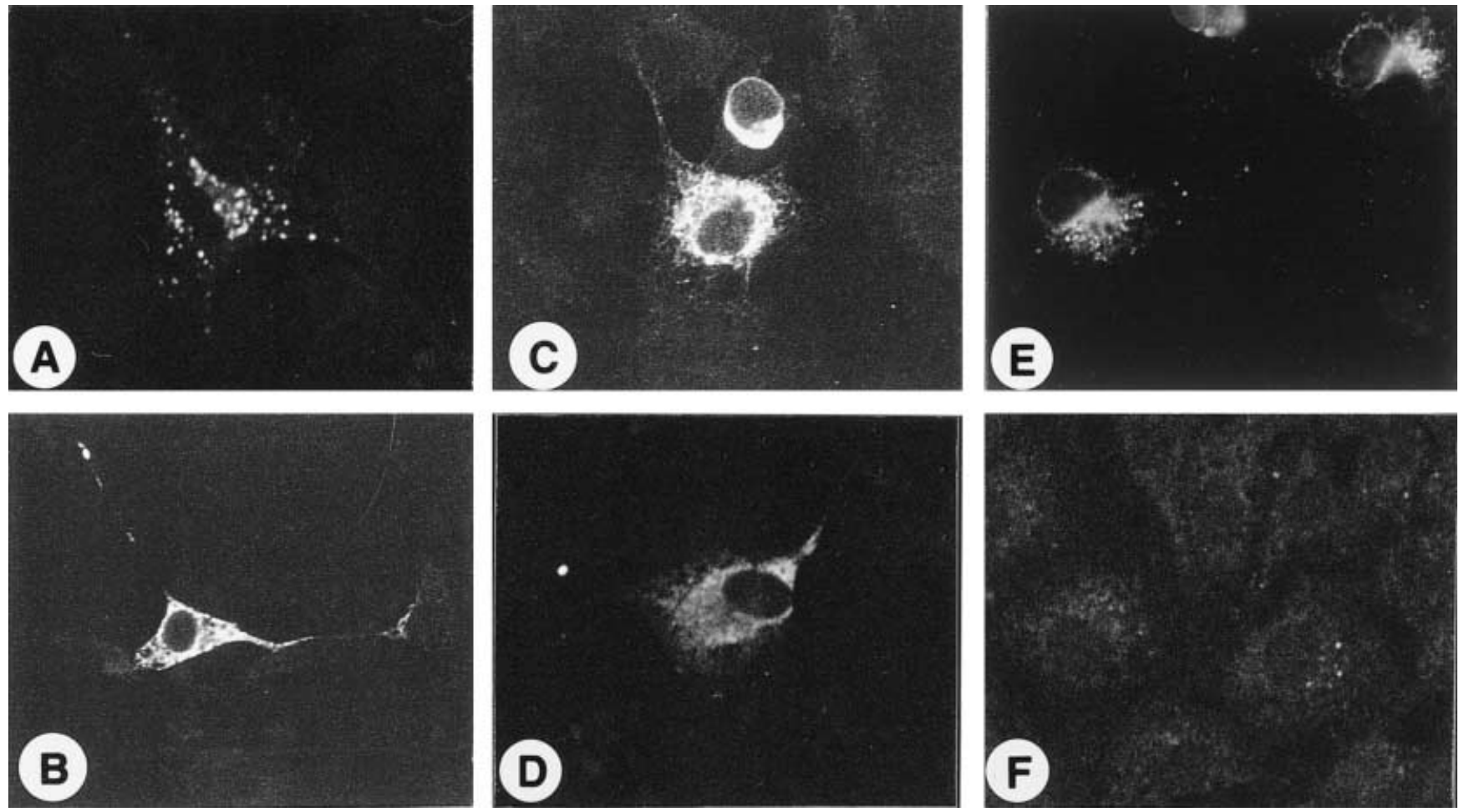

\section{x 400}

Fig. 1. Immunocytochemical analysis of lysosomal sialidase expressed in COS-1 cells. A cotransfected with the wild-type lysosomal sialidase (Sial) cDNA and PPCA cDNA. B cotransfected with the Sial cDNA containing the P80L mutation and PPCA cDNA. C cotransfected with the Sial cDNA containing the W240R mutation and PPCA cDNA

P80L. The P80 residue is situated in a conserved (F)RIP motif, which is at the $\mathrm{N}$-terminus of the first strand of the first $\beta$-sheet unit. The side chain of the P80 residue is adjacent to the active site residue R78, as well as to Y370 and E394, which are located in the first strand of the sixth $\beta$ sheet unit in the wild-type structure. As shown in Fig. 3 (left), the amino acid substitution of P80 to L caused conformational changes of the main chain that affected the position of the active site residues R78, Y370, and E394.

P316S. P316 is localized in the N-terminal region of the first strand of the fifth $\beta$-sheet unit, and is adjacent to the active site residues R280 and Y370. Fig. 3 (right) shows that the substitution of $\mathrm{P} 316$ to $\mathrm{S}$ caused a conformational change of the main chain of the fifth $\beta$-sheet unit containing P316 as well as the R280 and Y370 residues adjacent to P316.

W240R. W240 is situated in the conserved Asp box between the third and fourth strands of the third $\beta$-sheet unit. The side chain of the W240 residue is packed on the inner side of the modeled structure of wild-type sialidase (Fig. 2). In contrast, the transversion to R240 is predicted to cause the side chain to come to the surface of the molecule. It may also alter the conformation of the fourth strand of the second $\beta$-sheet unit as well as of the fourth strand of the third unit. These structural changes are localized on the surface

D cotransfected with the Sial cDNA containing the P316S mutation and PPCA cDNA. E cotransfected with the Sial cDNA containing the V217M mutation and PPCA cDNA. F mock transfection with PPCA cDNA. $\times 400$

of the sialidase molecule near the W240 residue (Fig. 4, W240R).

G243R. G243 is located on the molecular surface of the wild-type sialidase. Substitution to R243 influences the conformation of the fourth strand of the third $\beta$-sheet and the loop structure connecting the third and fourth strands of the fourth $\beta$-sheet unit (Fig. 2), located on the molecular surface near the G243 residue (Fig. 4, G243R).

$V 217 M$. V217 is present in the N-terminal region of the second strand of the third $\beta$-sheet unit, which is located on the lower and inner side of the wild-type sialidase molecule (Fig. 2). The molecular surface region altered by transversion of V217 to $\mathrm{M}$ is deduced to be overlapped by those altered as the result of the W240R and G243R substitutions. However, the alterations are smaller than those caused by the W240R and G243R changes (Fig. 4, V217M).

\section{Discussion}

In the present study, three novel missense mutations $(\mathrm{P} 80 \mathrm{~L}$, W240R, and P316S) were identified in the coding region of the human lysosomal sialidase gene in two Japanese 
Fig. 2. Modeled three-dimensional structure of the wild-type human lysosomal sialidase. The secondary structures are shown as a tube and ribbon drawing; $\alpha$ helix (red), $\beta$-sheet (blue), and coil (yellow). The active site residues (R78, R97, D103, D135, S156, E264, R280, Q282, R341, $Y 370$, and E394) are shown in magenta. The locations of mutations (P80, V217, W240, G243, and $P 316)$ are shown in cyan

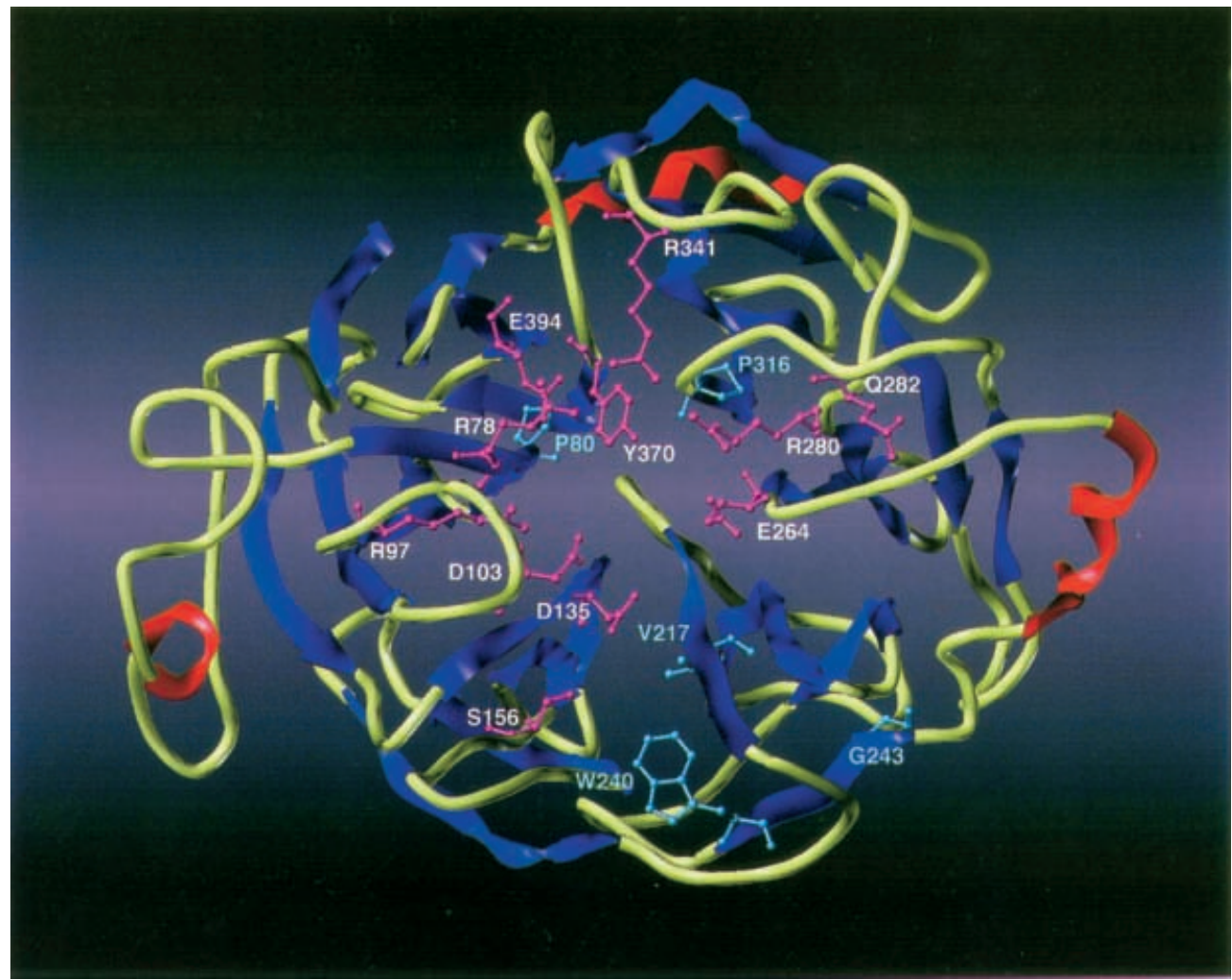

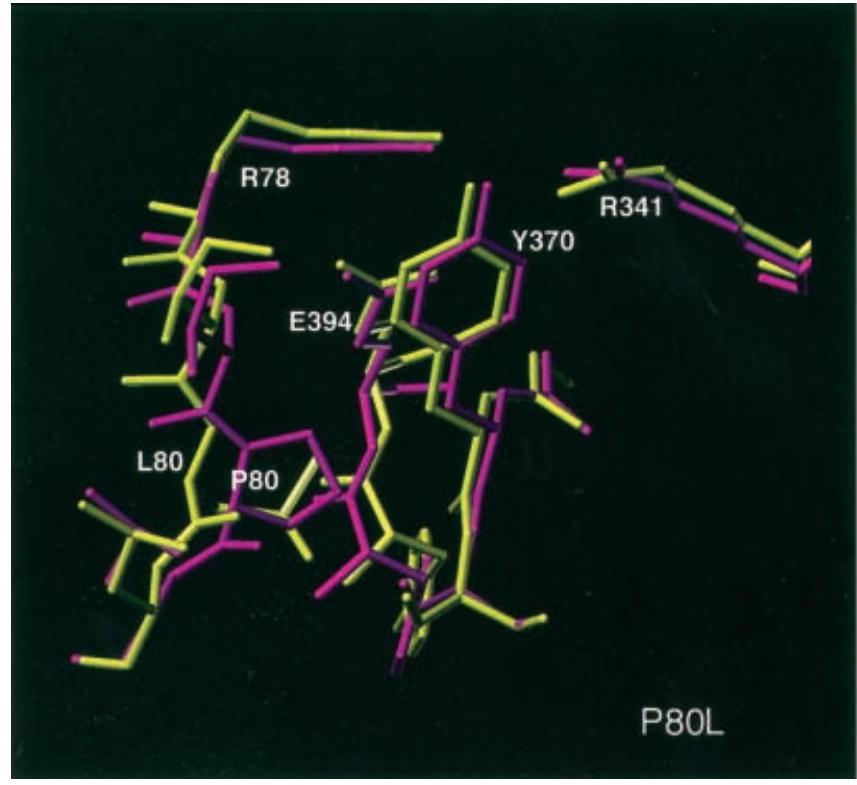

Fig. 3. Conformational changes caused by the P80L and P316S mutations. The residues of the wild-type and mutant enzymes are colored in magenta and yellow, respectively. The residues influenced by the re-

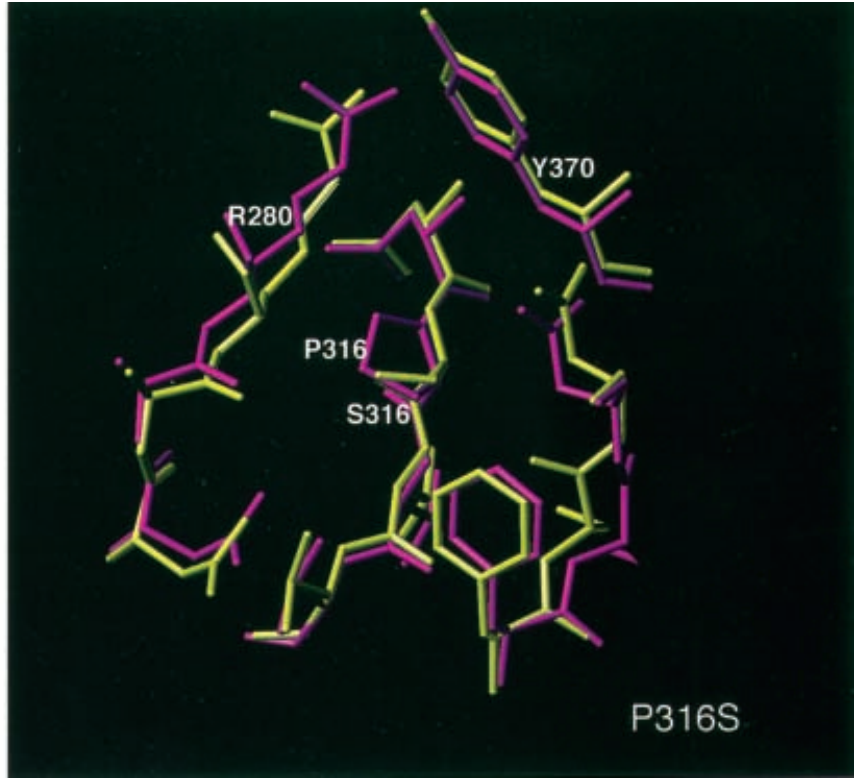

placement of $P 80$ with $L$ are shown on the left $(R 78, R 341, Y 370$, and E394), and those influenced by replacement of $P 316$ with $S$ on the right (R280 and Y370) sialidosis patients. A patient with a severe, congenital type 2 phenotype was a compound heterozygote with mutant alleles causing the amino acid substitutions P80L and W240R. The other patient, whose phenotype is considered to be type 1 because of its relatively late onset and mild manifestations, was a homozygote for the mutant allele causing the P316S change. The expression studies revealed that none of the mutant cDNA products showed enzyme activity toward 4-methylumbelliferyl $N$-acetyl neuraminic acid, or had a typical lysosomal distribution in cultured cells. Because the new mutant products were hardly detected by immunoblotting (data not shown), they were 

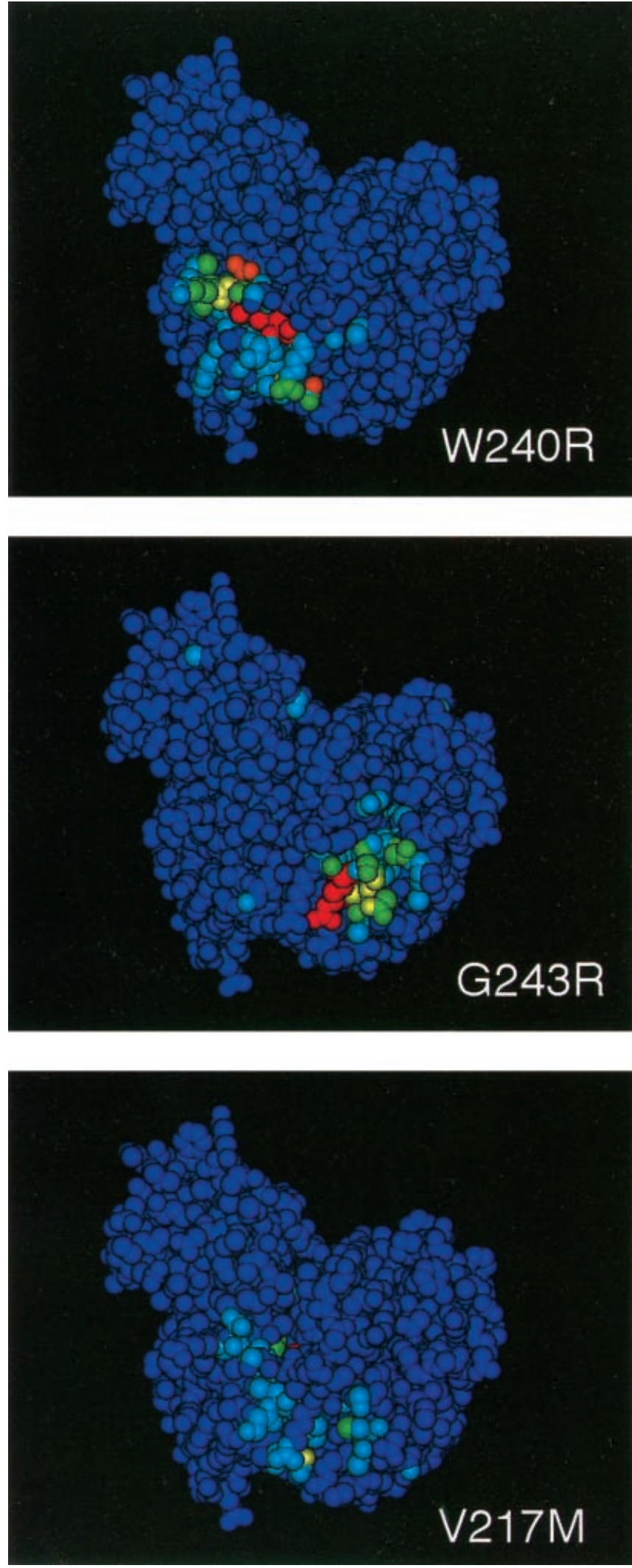

Fig. 4. Structural changes on the molecular surface caused by point mutations $W 240 R, G 243 R$, and V217M. Each deduced mutant structure is shown as a space-filled model. After superimposing the mutan and wild-type structures, each atom was colored according to the distance between the atom in the mutant and the corresponding atom in the wild-type structure. The colors of the atoms show the distance ranges as follows: blue $<0.5 \AA, 0.5 \AA \leqq$ cyan $<1.0 \AA, 1.0 \AA$ green $<$ $1.5 \AA, 1.5 \AA \leqq$ yellow $<2.0 \AA, 2.0 \AA$ orange $<2.5 \AA$, red $\geqq 2.5 \AA$ considered to not show any important difference in intracellular stability. Previously, we identified two missense mutations causing the amino acid substitutions V217M and G243R in two unrelated Japanese patients with type 1 sialidosis (Naganawa et al. 2000). In cultured galactosialidosis fibroblastic cells expressing the G243R mutant cDNA, neither sialidase activity nor lysosomal localization was observed. In contrast, residual enzyme activity and partial lysosomal localization of the gene product were observed in the cells expressing the V217M mutant. The latter mutation was considered to cause a mild sialidosis phenotype (Lowden and O'Brien 1979; Thomas 2000). In the present study, the gene products with P80L and W240R substitutions identified in the clinically severe patient as well as that with the P316S substitution identified in the late-onset case did not exibit any enzyme activity toward an artifical substrate or affect the intracellular distribution of the wild-type lysosomal sialidase. The reason why the homozygous patient with the P316S mutation shows a milder clinical phenotype is not clearly understood at present. One possible explanation is that a small part of the P316S mutant product may be translocated to lysosomes where it is rapidly degraded. Another possibility is that the P316S products may retain residual sialidase activity toward the natural substrates in vivo.

The molecular modeling predicted that the P80L and P316S substitutions could alter the conformation including the active site residues R78, R280, R341, Y370, and E394. For example, the P80L change influences the active site residues R78, R341, Y370, and E394. On the other hand, the P316S substitution can influence the R280 and Y370 residues. Crennell et al. (1994) reported the functional roles of active site residues on sialidases of bacterial/viral origin on the basis of the structures of their complexes with inhibitors (Burmeister et al. 1992; Crennell et al. 1993, 1994; Gaskell et al. 1995). They suggested that the active site arginine residues corresponding to residues R78, R280, and R341 of the human sialidase contribute to stabilization of the carbonic acid residue commonly present in all sialic acid substrates. The hydroxy groups of tyrosine residues corresponding to residue $\mathrm{Y} 370$ of the human enzyme might be located near the carbon atoms $(\mathrm{C} 1$ and $\mathrm{C} 2)$ of sialic acid, and the glutamic acid residues corresponding to E394 might stabilize R78. Accordingly, the degree of the defect in substrate recognition or hydrolysis caused by the P316S transversion may be smaller than that caused by the P80L substitution. This may account for the milder phenotype of the homozygous sialidosis patient with the P316S substitution, although the progression of the symptoms in this patient is more rapid than that in the patients with the V217M change, as previously reported (Naganawa et al. 2000).

Another group of substitutions including W240R, G243R, and V217M are deduced to cause structural alterations in a restricted surface region of the sialidase model. Lukong et al. (2000) reported that the surface structure close to the present region is influenced by the A298V substitution. For comparison with these results, we also constructed a mutant model with the A298V substitution (data not shown). We observed that all three substitutions 
could cause structural changes in similar regions of the sialidase surface. The conformational changes on the sialidase surface induced by the V217M mutation were smaller than those in the case of W240, G243R, or A298V. Taken together with the findings that the V217M mutant gene product partially retains sialidase activity and the ability to be translocated to lysosomes, the surface structure of this region may be essential for the translocation of sialidase to lysosomes, which ensures the full expression of the enzyme activity. Lukong et al. (2000) also speculated that this surface area contributes to the molecular interaction with other components of a multienzyme complex, PPCA and/or $\beta$-galactosidase. Recently, Bonten et al. (2000) reported gene mutations, some of them novel, causing amino acid substitutions in eleven sialidosis patients. The expressed enzymes with amino acid substitutions were characterized and assigned to three groups on the basis of their subcellular distribution and residual catalytic activity. One group (G218A, L231H, P335Q, and L363P) was catalytically inactive and not lysosomal. The second group (G227R, F260Y, and Y370C) was catalytically inactive but lysosomal. The third group (V54M, R294S, G328S, and dpl399HY) was partially active and lysosomal. They also mentioned a close correlation between the residual activity of the mutant gene products and the clinical severity of the disease. It is interesting that many of the amino acid substitutions (for example, G218A, L231H, and G227R) causing relatively severe clinical phenotypes are localized around the restricted molecular surface regions as deduced in this study. These regions also contain other mutations reported by Lukong et al. (2000). Further structural analysis will allow more informative predictions and correlations between structures and biochemical characteristics of the mutant enzymes.

Acknowledgments This research was supported by grants from the Japan Society for the Promotion of Science, the Ministry of Health, Labor and Welfare of Japan, and the Tokyo Metropolitan Government.

\section{References}

Bonten E, d'Azzo A (2000) Lysosomal neuraminidase. J Biol Chem 275:37657-37663

Bonten E, van der Spoel A, Fornerod M, Grosveld G, d'Azzo A (1996) Characterization of human lysosomal neuraminidase defines the molecular basis of the metabolic storage disorder sialidosis. Genes Dev 10:3156-3169

Bonten E, Arts WF, Beck M, Covanis A, Donati MA, Parini R, Zammarchi E, d'Azzo A (2000) Novel mutations in lysosomal neuraminidase identify functional domains and determine clinical severity in sialidosis. Hum Mol Genet 9:2715-2723

Burmeister WP, Ruigrok RWH, Cusack S (1992) The 2.2 A resolution crystal structure of influenza $\mathrm{B}$ neuraminidase and its complex with sialic acid. EMBO J 11:49-56

Claessens M, van Cutsem E, Lasters I, Wodak S (1989) Modeling the polypeptide backbone with "spare parts" from known protein structures. Protein Eng 2:335-345

Crennell SJ, Garman EF, Laver WG, Vimr ER, Taylor GL (1993) Crystal structure of a bacterial sialidase (from Salmonella typhimurium LT2) shows the same fold as an influenza virus neuraminidase. Proc Natl Acad Sci U S A 90:9852-9856
Crennell SJ, Garman EF, Laver WG, Vimr ER, Taylor GL (1994) Crystal structure of a Vibrio Cholerae sialidase reveals dual lectinlike domains in addition to the catalytic domain. Structure 2:535544

Davis LG, Dibner MD, Battey JF (1986) In: Basic methods in molecular biology. Elsevier, New York

d'Azzo A, Hoogeveen A, Reuser AJ, Robinson D, Galjaard H (1982) Molecular defect in combined beta-galactosidase and neuraminidase deficiency in man. Proc Natl Acad Sci U S A 79:4535-4539

d'Azzo A, Andria G, Strisciuglio P, Galjaard H (2000) Galactosialidosis. In: Scriver CR, Beaudet AL, Sly WS, Valle D (eds) The metabolic and molecular bases of inherit disease, 8th edn. McGrawHill, New York, pp 3811-3826

Gaskell A, Crennel SJ, Taylor GL (1995) The three domains of a bacterial sialidase: a $\beta$-propeller, an immunoglobulin module and a galactose-binding jelly-roll. Structure 3:1197-1205

Gluzman Y (1988) SV40-transformed simian cells support the replication of early SV40 mutants. Cell 23:175-182

Guarnieri FG, Arterburn LM, Penno MB, Cha Y, August JT (1993) The motif Tyr-X-X-hydrophobic residue mediates lysosomal membrane targeting of lysosome-associated membrane protein 1. J Biol Chem 268:1941-1946

Hiraiwa M, Nishizawa M, Uda Y, Nakajima T, Miyatake T (1988) Human placental sialidase: further purification and characterization. J Biochem 103:86-90

Itoh K, Takiyama N, Nagao Y, Oshima A, Sakuraba H, Potier M, Suzuki Y (1991) Acid carboxypeptidase deficiency in galactosialidosis. Jpn J Hum Genet 36:171-178

Jones TA, Thirup S (1986) Using known substructures in protein model building and crystallography. EMBO J 5:819-822

Kabsh W (1976) A solution for the best rotation to relate two sets of vectors. Acta Crystallogr A32:922-923.

Kabsh W (1978) A discussion of the solution for the best rotation to relate two sets of vectors. Acta Crystallogr A34:827-828

Lowden J, O'Brien J (1979) Sialidosis: a review of human neuraminidase deficiency. Am J Hum Genet 31:1-18

Lukong KE, Elsliger M-A, Chang Y, Richard C, Thomas G, Carey W, Tylki-Szymanska A, Czartoryska B, Buchholz T, Criado GR, Palmeri S, Pshezhetsky AV (2000) Characterization of the sialidase molecular defects in sialidosis patients suggests the structural organization of the lysosomal multienzyme complex. Hum Mol Genet 9:1075-1085

Milner CM, Smith SV, Carrillo MB, Taylor GL, Hollinshead M, Campbell RD (1997) Identification of a sialidase encoded in the human major histocompatibility complex. J Biol Chem 272:45494558

Naganawa Y, Itoh K, Shimmoto M, Takiguchi K, Doi H, Nishizawa Y, Kobayashi T, Kamei S, Lucong KE, Pshezhetsky AV, Potier M, Sakuraba H (2000) Molecular and structural studies of Japanese patients with sialidosis type I. J Hum Genet 45:241-249

Pshezhetsky AV, Richard C, Michaud L, Igdloura S, Wang S, Elsliger M-A, Qu J, Leclerc D, Dallaire L, Potier M (1997) Cloning, expression and chromosomal mapping of human lysosomal sialidase and characterization of mutations in sialidosis. Nat Genet 15:316320

Roggentin P, Rothe B, Kaper JB, Galen J, Lawrisuk L, Vimr ER, Schauer R (1989) Conserved sequences in bacterial and viral sialidases. Glycoconj J 6:349-353

Rothe B, Rothe B, Roggentin P, Schauer R (1991) The sialidase gene from Clostridium septicum: cloning, sequencing, expression in Escherichia coli and identification of conserved sequences in sialidases and other proteins. Mol Gen Genet 226:190-197

Sasagasako N, Miyahara S, Saito N, Shinnoh N, Kobayashi T, Goto I (1993) Prenatal diagnosis of congenital sialidosis. Clin Genet 44:8-11

Shimmoto M, Fukuhara Y, Itoh K, Oshima A, Sakuraba H, Suzuki Y (1993) Protective protein gene mutations in galactosialidosis. J Clin Invest 91:2393-2398

Sosa MA, Schmidt B, von Figura K, Hille-Rehfeld A (1993) In vitro binding of plasma membrane-coated vesicle adaptors to the cytoplasmic domain of lysosomal acid phosphatase. J Biol Chem 268: $12537-12543$

Sussman JL, Lin D, Jiang J, Manning NO, Prilusky J, Ritter O, Abola EE (1998) Protein Data Bank (PDB): database of three-dimensional structural information of biological macromolecules. Acta Crystallogr D54:1078-1084 
Sutcliffe MJ, Hayes FRF, Blundell TL (1987) Knowledge-based modeling of homologous proteins, part II: rules for conformations of substituted side chains. Protein Eng 1:385-392

Suzuki K (1987) Enzymatic diagnosis of sphingolipidoses. In: Ginsbury V (ed) Methods in enzymology, vol 138. Academic, New York, pp 727-762

Suzuki Y, Sakuraba H, Potier M, Akagi M, Sakai M, Beppu H (1981) $\beta$-Galactosidase-neuraminidase deficiency in adults: deficiency of a freeze-labile neuraminidase in leukocytes and fibroblasts. Hum Genet 58:387-389

Thomas GH (2000) Disorders of glycoprotein degradation: $\alpha$ mannosidosis, $\beta$-mannosidosis, fucosidosis, and sialidosis. In: Scriver CR, Beaudet AL, Sly WS, Valle D (eds) The metabolic and molecular bases of inherited disease, 8th edn. McGraw-Hill, New York, pp 3507-3534

van der Horst G, Galjart AT, d'Azzo A, Galjaard H, Verheijen FW (1989) Identification and in vitro reconstitution of lysosomal neuraminidase from human placenta. J Biol Chem 264:13171322

van der Spoel A, Bonten EJ, d'Azzo, A (1998) Transport of human lysosomal neuraminidase to mature lysosomes requires protective protein/cathepsin A. EMBO J 17:1588-1597

Verheijen FW, Brossmer R, Galjaard H (1982) Purification of acid $\beta$ galactosidase and acid neuraminidase from bovine testis: evidence for an enzyme complex. Biochem Biophys Res Commun 108:868875

Verheijen FW, Palmeri S, Hoogeveen AT, Galjaard H (1985) Human placental neuraminidase. Activation, stabilization and association with $\beta$-galactosidase and its protective protein. Eur J Biochem 149:315-321

Vinogradova MV, Michaud L, Mezentsev AV, Lukong KE, El-Alfy M, Morales CR, Potier M, Pshezhetsky AV (1998) Molecular mechanism of lysosomal sialidase deficiency in galactosialidosis involves its rapid degradation. Biochem J 330:641-650 